\title{
Structure analysis of twinned crystals of tris(N,N-di-n-butyldiselenocarbamato)nickel(IV) bromide
}

\author{
P. T. Beurskens And J. A. Cras \\ Department of Inorganic Chemistry, University of Nijmegen, \\ Nijmegen, The Netherlands \\ (Received 28 January 1970)
}

Abstract

The crystal structure of tris(N,N-di- $n$-butyldiselenocarbamato)nickel(IV) bromide, $\mathrm{Ni}\left(\mathrm{Se}_{2} \mathrm{CN}\right.$ $\left.\left(\mathrm{C}_{4} \mathrm{H}_{9}\right)_{2}\right)_{3} \mathrm{Br}$, has been determined by three-dimensional X-ray methods. The Laue symmetry is $6 / \mathrm{mmm}$, but the structure could be solved only in space group $P 31 c$. The crystals were, therefore, regarded as being microtwinned on (001). The unit cell dimensions are $a=b=14.69$ (3) and $c=$ 10.91(3) \&. From integrated Weissenberg photographs, 307 non-zero symmetry-independent reflexions were measured photometrically. The structural parameters were refined by least-squares methods to a conventional $R$ factor of $0 \cdot 10$.The quadrivalent nickel atom is octahedrally coordinated by six selenium atoms. The $\mathrm{Ni}-\mathrm{Se}$ distance is 2-391(5) $\AA$; the Se-Se distance within one diselenocarbamate ligand is 3.00(1) $\AA$; Se-Se distances between different ligands are 3.51(1), 3.52(1) and 3.55(1) A.

\section{Introduction}

Nickel(IV) complexes with sulphur-containing bidentate ligands are known (MacCleverty, 1968) to contain nickel atoms in square-planar coordination. The compounds tris( $N, \mathbf{N}$-di-n-butyldithiocarbamato)nickel(IV) bromide ( $\mathrm{Ni}(\mathrm{dtc})_{3} \mathrm{Br}$ ) and the isostructural seleno compound $\left(\mathrm{Ni}(\mathrm{dsc})_{3} \mathrm{Br}\right)$, reported in a previous paper (Brinkhoff et al., 1969), were expected to contain nickel(IV) in octahedral coordination. This has been confirmed by the present $\mathrm{X}$-ray analysis. As the sulphur compound did not give suitable crystals, the structure of the seleno compound $\left(\mathrm{Ni}(\mathrm{dsc})_{3} \mathrm{Br}\right)$ was investigated.

\section{Crystal data}

2. Tris(N,N-di- $n$-butyldiselenocarbamato)nickel(IV) bromide, $\mathrm{Ni}\left(\mathrm{Se}_{2} \mathrm{CN}\left(\mathrm{C}_{4} \mathrm{H}_{9}\right)_{2}\right)_{3} \mathrm{Br}, F W 1033 \cdot 13$; $F(000)=1048$. Black crystals, hexagonal prismatic $\{100\}$, elongated along $c$.

Trigonal; unit cell dimensions (with standard deviations), $a=b=14.69$ (3) and $c=10.91$ (3) $\AA$ (obtained from Weissenberg and oscillation photographs), $V=2037(10) \AA^{3} . D_{m t}=1.61(2) \mathrm{g} \mathrm{cm}^{-3}$ (pycnometer), $Z=2, D_{x}=1.55 \mathrm{~g} \mathrm{~cm}^{-3}$. Nickel-filtered copper radiation (Cu $K \alpha, \lambda=1.5418 \AA$ ); linear absorption coefficient, $\mu=86.9 \mathrm{~cm}^{-1}$. The diffraction pattern is hexagonal (Laue symmetry . $6 / \mathrm{mmm}$ ) with systematic absences $h h l$ for $l=2 n+1$.

Intensities of 307 non-zero $h k l$ reflexions were measured from nine layers of integrated Weissenberg photographs, using an optical densitometer. The crystal was small enough ( $0.04 \mathrm{~mm}$ diameter) to neglect an absorption correction. The three-dimensional Patterson synthesis could not be solved unless it was assumed that the crystals were microtwinned on (001). The structure was solved in the space group $P \overline{3} 1 c$, No. 163 (Laue symmetry $\overline{3} m$ ). 
Determination of the structure

Assuming the structure to be trigonal, the positions of the selenium could be found from the Patterson analysis. Several spacegroups were used for trials, but only one $P \overline{3} 1 c$, revealed the positions of the bromine and nickel atoms and allowed consequent refinement of the trial structure. The carbon and nitrogen atoms were found from difference Fourier syntheses. At this stage of the analysis, the unobserved reflexions were rejected; practically all had calculated intensities equal to or less than the minimum observable value.

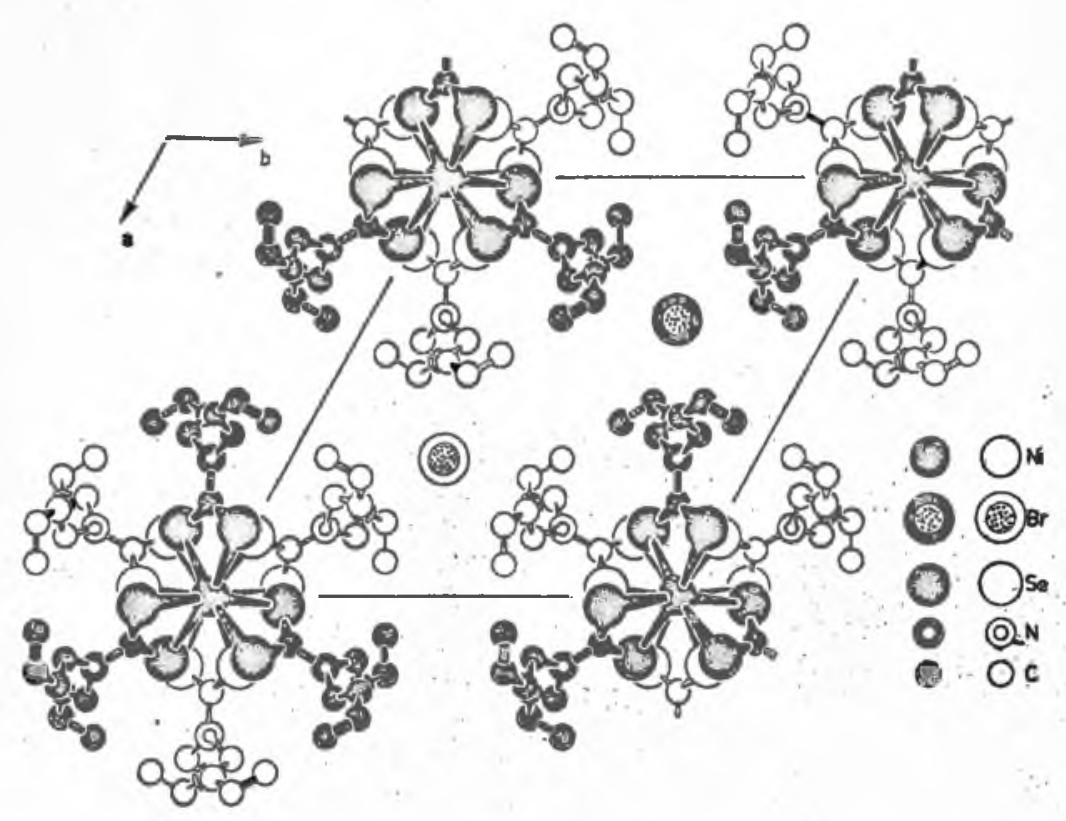

Fig. 1. Projection of $\mathrm{Ni}(\mathrm{dsc})_{3} \mathrm{Br}$ along $c$. The black ions are situated at $z=\frac{1}{4}$ and the white ions at $z=-1$. (The last carbon atom of the butyl chain is disordered, and can be found in several positions).

$$
\text { i. 'rumide, }
$$

Because of the twinning, the measured intensities of the $h k l$ reflexions are actually the rms values of the $h k l$ and $h k l$ intensities. A least-squares programme was, therefore, written to minimize the function

$$
\sum_{h k l} w\left[F_{o}(h k l)-\left(\frac{1}{2} F_{c}^{2}(h k l)+\frac{1}{2} F_{c}^{2}(h k l)\right)^{1 / 2}\right]^{2}
$$

with weight $w=1 /\left(60+F_{o}+0.06 F_{o}^{2}\right)$.

Full-matrix refinement of all the positional and isotropic temperature-factor parameters and the nine layer scale factors gave $\boldsymbol{R}=0.096$. From a final difference. Fourier synthesis, it became apparent that the last carbon atom of the butyl chain is disordered and can be found in several positions. Atomic parameters are given in Table 1, observed and calculated structure factors in Table 2 and distances and angles in Table 3. 
STRUCTURE ANALYSIS OF TWINNED CRYSTALS

Table 1. Positional and isotropic vibration parameters

\begin{tabular}{lllll}
\hline & \multicolumn{1}{c}{$\boldsymbol{x}$} & \multicolumn{1}{c}{$y$} & \multicolumn{1}{c}{$z$} & $\boldsymbol{B}\left(\AA^{2}\right)$ \\
\hline $\mathrm{Ni}$ & 0.0000 & 0.0000 & 0.0000 & $2 \cdot 9(3)$ \\
$\mathrm{Se}$ & $0-0190(3)$ & $0 \cdot 1464(3)$ & $0.1356(5)$ & $2 \cdot 9(3)$ \\
$\mathrm{C}(1)$ & $0 \cdot 111(2)$ & $0.222(4)$ & 0.2500 & $2 \cdot 1(9)$ \\
$\mathrm{N}$ & $0.168(2)$ & $0.336(4)$ & 0.2500 & $3 \cdot 9(9)$ \\
$\mathrm{Br}$ & 0.3333 & 0.6667 & 0.2500 & $4 \cdot 4(3)$ \\
\hline
\end{tabular}

The estimated standard deviations, in parentheses, are obtained from the least-squares programme. The parameters of the butyl chain and the anisotropic parameters of selenium are omitted because of their limited physical significance.

\section{Description of the structure}

The structure (Figs. 1 and 2) contains tris(N,N-di-n-butyldiselenocarbamato)nickel(IV)ions, $\mathrm{Ni}(\mathrm{dsc})_{3}{ }^{+}$. They have point group symmetry $32\left(D_{3}\right)$, and are piled along the threefold axis, adjacent ions being related by a centre of symmetry. The bromide ions, $\mathrm{Br}^{-}$, are located in the holes formed by the butyl chains.

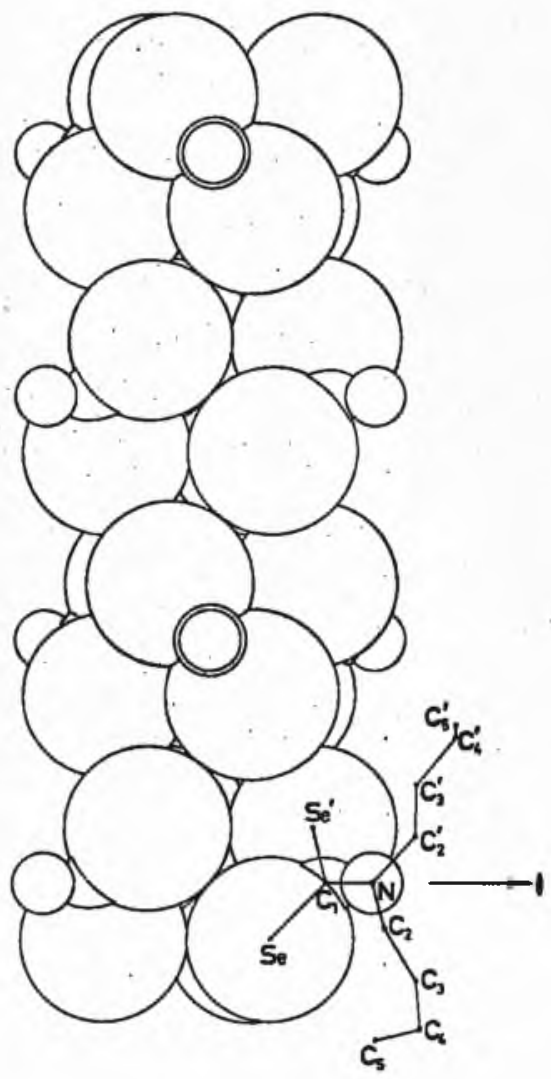

Fig. 2. Drawing of $\mathrm{Ni}(\mathrm{dsc})_{3}{ }^{+}$ions, piled along $c$. The primed atoms are related to the unprimed atoms by a two-fold axis. The butyl chains are omitted except for oneligand. (The nickel atoms are not visible.) 
Table 2. Observed and calculated structure factors.

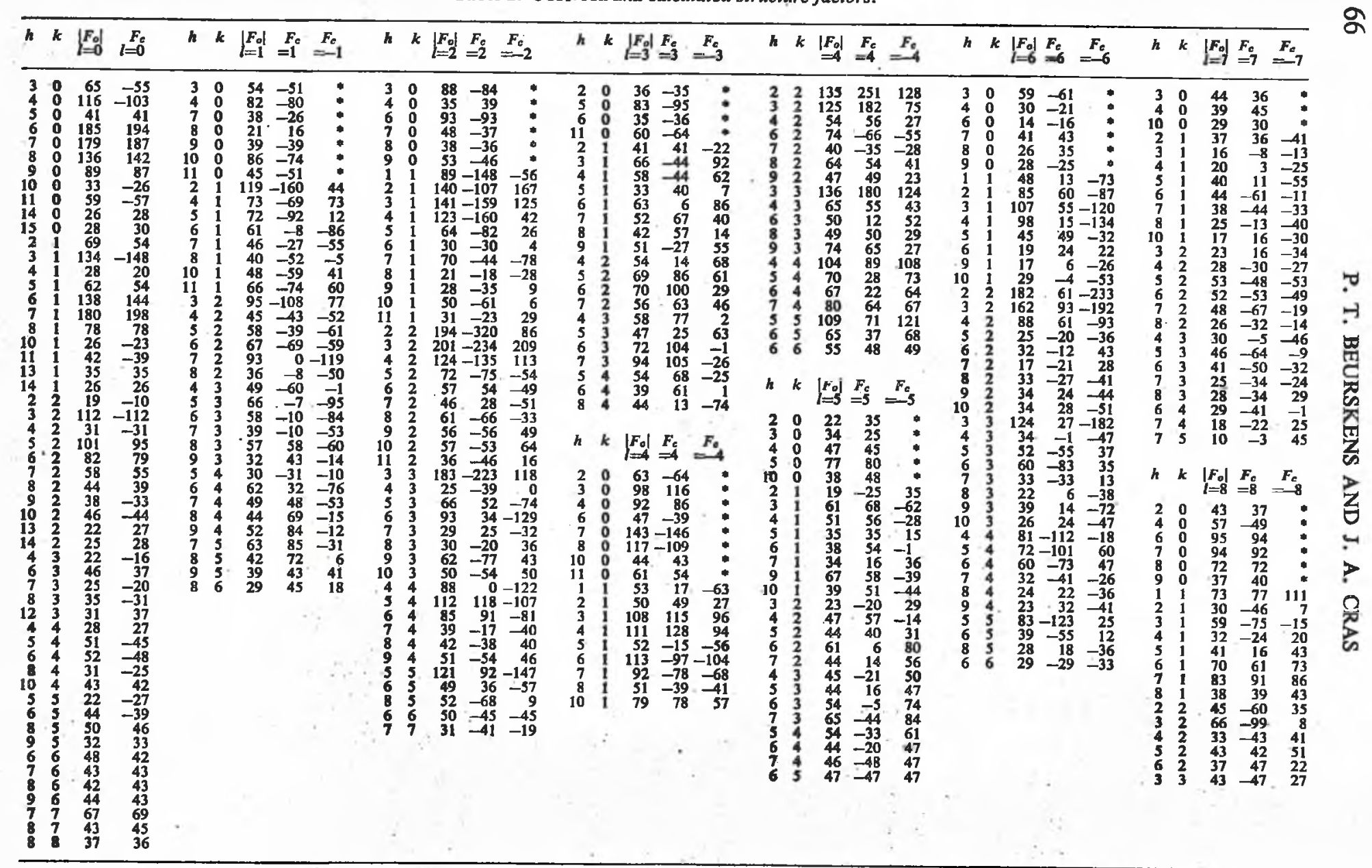

For each observed reflexion $F_{c}(h k l)$ and $F_{c}(h k l)$ are listed, unless they are related by symmetry. In view of the twinning, the observed structure factor $\left|F_{0}(h k l)\right|$ is supposed to be the observed value for the root mean squares of $F_{c}(h k l)$ and $F_{c}(h k l)$. 
The Ni-Se bond, $2 \cdot 391(5) \AA$, is slightly longer, and the $\mathrm{Se}-\mathrm{Ni}-\mathrm{Se}$ angle, $77 \cdot 7(4)^{\circ}$, is more acute, than in bis (di-ethyldiselenocarbamato)nickel(II) (2.317 $\AA$ and $81^{\circ}$, respectively) in which nickel is in planar four-fold coordination (Bonamico \& Dessy, 1967). This is contrary to expectations if one considers the oxidation states of the nickel atom, but is to be expected if one assumes the packing to be controlled by the normal Se-Se van der Waals distance, $3.53 \AA$ (compare the values quoted in Table 3).

Table 3. Distances and angles

\begin{tabular}{|c|c|c|c|}
\hline $\begin{array}{l}\mathrm{Ni}-\mathrm{Se} \\
\mathrm{Se}-\mathrm{Se}^{\prime} \\
\mathrm{Se}-\mathrm{Se}^{\prime \prime} \\
\mathrm{Se}-\mathrm{Se}^{\prime \prime} \\
\mathrm{Se}-\mathrm{C}(1) \\
\mathrm{C}(1)-\mathbf{N}\end{array}$ & $\begin{array}{l}2 \cdot 391(5) \AA \\
3 \cdot 00(1) \AA \\
3 \cdot 51,3 \cdot 52(1) \AA \\
3 \cdot 55(1) \AA \\
1 \cdot 78(3) \AA \\
1 \cdot 48(8) \AA\end{array}$ & $\begin{array}{l}\mathrm{Se}-\mathrm{Ni}-\mathrm{Se}^{\prime} \\
\mathrm{Se}-\mathrm{Ni}-\mathrm{Se}^{\prime \prime} \\
\mathrm{Se}-\mathrm{C}(1)-\mathrm{Se}^{\prime}\end{array}$ & $\begin{array}{l}77 \cdot 4(4)^{\circ} \\
94 \cdot 4,94 \cdot 7(4)^{\circ} \\
114(1)^{\circ}\end{array}$ \\
\hline
\end{tabular}

$\mathrm{Se}^{\prime}$ refers to selenium in the same ligand.

$\mathrm{Se}^{\prime \prime}$ refers to selenium in other ligands of the cation.

Se" refers to selenium in adjacent cations.

We thank Professor J. J. Steggerda for his continuous interest, and Mr J. M. M. Smits for valuable assistance.

\section{References}

Bonamico, M. \& Dessy, G. (1967) Chem. Comm. 21, 1114 (corrected, private communication). Brinkhoff, H. C., Cras, J. A., Steggerda, J. J. \& Willemse, J. (1969) Rec. Trav. Chim. 88, 633.

MacCleverty, J. A., Progress in Inorganic Chemistry, Vol. X, ed. Cotton, F. A. (Interscience, 1968, pp. 49-221). 